\title{
O $\mathbb{M} \mathbb{A} R \mathbb{R} \mathbb{R} \mathbb{T} \mathbb{N} G \mathbb{I} \mathbb{T} \mathbb{R} \mathbb{R} O \mathbb{C O M}$

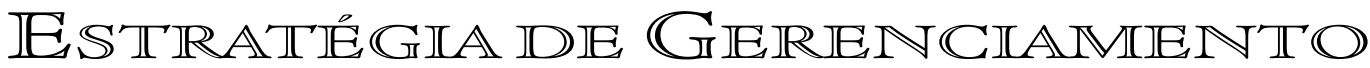 \\ DOS RECURSOS $\mathbb{H} \mathbb{R} U \mathbb{M} \mathbb{A}$ NOS $\mathbb{E} \mathbb{M}$ \\ IIOTÉIS: UNM ESTUDO DE CASO NA

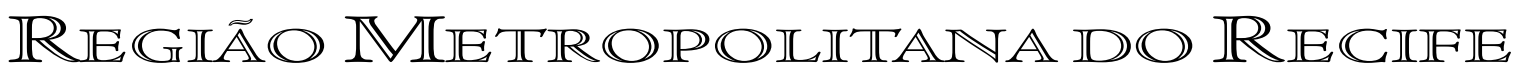

\author{
Maria de Lourdes de Azevedo Barbosa* \\ Lúcia Maria Barbosa de Oliveira**
}

RESUMio

O

presente estudo tem como objetivo analisar se a gestão de recursos humanos adotada por um dos hotéis representativo da rede hoteleira da cidade do Recife (PE) contribui para alcançar a qualidade na prestação dos serviços.

O trabalho tem um caráter exploratório e caracteriza-se como um estudo de caso. A coleta de dados foi realizada na empresa, através de pesquisa documental e entrevistas semi-estruturadas. Para avaliação dos resultados, utilizou-se o método de análise de conteúdo, tendo como referencial teórico à gestão de recursos humanos e ao marketing interno, que fornecem suporte à análise dos dados.

Como resultado, a pesquisa revelou que a gestão de recursos humanos, praticada pelo hotel estudado, apresenta-se consistente e bem estruturada, o que possibilita despertar e desenvolver no seu colaborador um sentido de compromisso com a qualidade na prestação de serviços hoteleiros, permitindo colocá-la numa condição de vantagem competitiva diante dos concorrentes. Os resultados do estudo vêem ao encontro do que foi discutido no referencial teórico relativo a gestão de recursos humanos e o marketing interno como forma de contribuir para o aumento da competitividade da organização.

$\mathbb{A B S T R A C T}$

his study aims at analysing whether the management of human resources adopted by one of the hotel which is representative of hospitality organizations in Recife may help it to achieve the quality of service rendering and hospitality.

The work has an exploratory focus and is characterised as a case study. The data have been collected through documentary research and semi-structured interviews. The technique known as content analysis was used to evaluate the results and the theoretical framework adopted was based on the human resource management and the internal marketing.

* Professora e Coordenadora do Curso de Hotelaria da Universidade Federal de Pernambuco.

** Professora e Coordenadora de Extensão e Pós-graduação da FBV - Faculdade Boa Viagem - Recife - PE; Profesora Participante do PROPAD - Programa de Pós-Graduação em Administração da Universidade Federal de Pernambuco. Pesquisadora doCNPq. 


\section{UMI PANORAMA DA INDÚSTRIA IIOTELEIRA}

partir da década de 80, nos países industrializados, e dos anos 90, no Brasil, as empresas passaram a enfrentar um novo desafio, pois as melhorias de eficiência promovidas nas atividades internas deixaram gradativamente de garantir sua sobrevivência e seu crescimento. O aumento da competição internacional pelos mercados impôs novos padrões de desempenho produtivo, tecnológico e mercadológico às empresas que pretendam alcançar um nível de competitividade global (BASSI,1997).

O mercado hoteleiro também sofre as conseqüências dessa nova ordem. No caso do Brasil, a estabilização da economia, em meados de 1994, marcou o início de um novo ciclo de investimentos em modernização e ampliação do parque hoteleiro nacional, que foram viabilizados pela entrada de novos agentes no setor, como os fundos institucionais e as incorporadoras imobiliárias, bem como os investimentos públicos em infra-estrutura turística, vinculados aos programas de geração de renda, como o PRODETUR/NE (Programa de Desenvolvimento do Turismo do Nordeste) e o BNDES (Banco Nacional de Desenvolvimento Econômico e Social), que a partir de 1991 passou a financiar projetos hoteleiros. Estes novos empreendimentos, devem consolidar um investimento na ordem de US\$ 5 bilhões entre 1997 e 2003 (PANORAMA SETORIAL, GAZETA MERCANTIL, 1999).

$O$ incremento no parque hoteleiro promoveu a expansão e a entrada de novas operadoras internacionais (Choice Atlântica, Renaissance/Marriot, Holliday Inn, dentre outras) o que vêm estimulando a reorganização do setor, através da definição de novos padrões de concorrência. Por essa razão, o período pode também ser marcado pela necessidade de profissionalização da administração dos hotéis e pelos investimentos em modernização e posicionamento de mercado dos empreendimentos estabelecidos (PANORAMA SETORIAL, GAZETA MERCANTIL, 1999).

Diante desse quadro expansionista e de acirramento da concorrência entre as empresas, a alternativa para a busca da competitividade parece estar centrada nos diferenciais, pois segundo PORTER (1994), as estratégias voltadas para a racionalização dos processos, melhorias na eficiência operacional e redução das estruturas, não geram diferenciais competitivos de longo prazo, pois podem ser facilmente copiados.

$\mathrm{Na}$ atividade hoteleira, os empregados podem estabelecer a diferença, pois eles "fazem parte do produto", na medida que os serviços são realizados num contexto em que produção, distribuição e consumo, ocorrem simultaneamente, com a presença quase que obrigatória do consumidor. Portanto, o hotel precisa ter uma equipe que atue bem durante os momentos de interação com o cliente (KOTLER et Al, 1999).

Como empresas prestadoras de serviços, os hotéis devem apostar, principalmente, na qualidade do elemento humano, já que a excelência do serviço, condição da competitividade e sobrevivência da empresa, depende de como ele está interagindo com os clientes. Correlação esta fácil de ser compreendida, mas nem sempre tão fácil de ser posta em prática, principalmente porque no Brasil, a hotelaria trabalha com excedentes de mercado, baixos salários e poucas exigências de especialização. De acordo com CARVALHO (1991), constitui-se junto com a construção civil, num mercado paralelo marginal, que absorve a todos, elevando, assim a rotatividade da atividade a índices por volta de $8 \%$ ao mês, com uma permanência média de 11 meses para $70 \%$ do quadro de funcionários de um hotel. Com isso, fica difícil de se manter padrões de serviço e controlar a qualidade. No que diz respeito aos profissionais mais especializados, continuam de hotel em hotel, procurando melhores condições de trabalho, salários, benefícios e qualidade de vida.

Parece claro, então, que a empresa hoteleira se depara constantemente com aspectos relativos às relações entre as pessoas e, conseqüentemente, à necessidade de gerenciar processos sociais, considerando-se as interações humanas como parte crucial do desempenho qualitativo do serviço oferecido. 
Portanto, tornar-se indispensável investir na qualidade e satisfação do próprio funcionário, a fim de que seja possível, para as empresas que atuam nesse segmento, alcançar um desempenho competitivo. Para que haja clientes satisfeitos, é preciso que haja empregados satisfeitos, afirma J. Willard Marriot, fundador da MARRIOT Corporation (COBRA E RANGEL, 1992).

Considerando-se que empregados fazem parte do produto hoteleiro, a empresa necessita ter uma equipe que atue bem, quando empregado e cliente estão em contato, já que aqueles que estão em contato direto com o cliente têm uma considerável responsabilidade pela provisão de um serviço de alta qualidade. Para tanto, é preciso que o marketing seja absorvido por todos os empregados, devendo fazer parte da filosofia da organização e ser sustentado, principalmente pelo pessoal de linha de frente.

O cliente interno torna-se um pilar importante para alcançar a qualidade dos serviços prestados e, nesse sentido, a área de recursos humanos torna-se fundamental e deve estar preparada para influenciar positivamente o bottom line da empresa, que corresponde aos aspectos relacionados à sobrevivência, crescimento, lucratividade, competitividade e flexibilidade da organização para adaptar-se às novas situações, por meio de atividades como o recrutamento, o treinamento, o retreinamento e a remuneração variável, dentre outros (SCHULER e HUBER, 1993).

De acordo com KOTLER et AL. (1999), a função de recursos humanos e do marketing são inseparáveis nas empresas hoteleiras, pois precisam trabalhar juntas a fim de criar tanto empregados como clientes satisfeitos.

Dentro desse contexto, surge uma considerável relação entre prestação de serviços, gestão de recursos humanos e o marketing interno. O equilíbrio e a interação entre estes elementos são valiosos para a satisfação dos clientes interno e externo e, conseqüentemente, para o resultado da empresa como um todo.

\section{A GESTÃO DE RECURSOS IIUMANOS NAS ORGANIZACGÓES MIODERNAS}

Nas organizações modernas, verifica-se um novo cenário a partir das transformações que vêm ocorrendo na economia global, principalmente no setor de serviços, em que se pode notar novas formas de trabalho, de estrutura e do valor atribuído aos recursos humanos.

Em nenhuma outra época na história de negócios e da indústria, as pessoas foram tão valorizadas como têm sido atualmente, e o que é mais notável é que essa valorização acentuada centra-se, essencialmente, no capital intelectual, na competência e no talento delas (MARQUADT \& ENGEL, 1993).

ULRICH (1998) considera que o ritmo acelerado das mudanças nos dias atuais, exigido pela globalização, tecnologia, crescimento lucrativo e demanda do consumidor, exerce grande influência nas organizações, colocando em pauta a competência da força de trabalho e as capacidades organizacionais.

Aliado a esses aspectos, GONÇALVES (1997) acrescenta que a empresa do século XXI será organizada em torno de seus processos e centrará seus esforços em seus clientes. Portanto, deverá ser ágil e enxuta, seus jobs (cargo, tarefa, emprego ou papel) exigirão conhecimento do negócio e autonomia, responsabilidade e habilidade na tomada de decisões.

As atividades concernentes à área de recursos humanos passam a ser, segundo ULRICH (1998), tanto operacionais quanto estratégicas. Precisam ser ao mesmo tempo polícia e parceiros. Assumir a responsabilidade tanto por metas qualitativas quanto quantitativas no curto e no longo prazo. Desempenhar papéis cada vez mais complexos e, às vezes, até mesmo paradoxais.

Novos paradigmas implicam em uma profunda modificação na forma tradicional de gerir pessoas nas organizações. Vencer as resistências internas às mudanças é o desafio gerencial a ser superado por aqueles que têm posições de liderança nas empresas (BOOG, 1994). 
Dentro desse contexto, GONÇALVES (1997) coloca que as organizações modernas passam a seguir novos princípios, tais como: alocação dinâmica de recursos, comunicação ponto a ponto, organização do trabalho em times e projetos, criação de valor, avaliação do desempenho por resultados, fronteiras orgânicas, oportunidade e coordenação, mais adequados à tecnologia e ao estilo gerencial contemporâneos.

Em função dos enormes desafios que se apresentam no contexto das organizações, cada vez mais, a gestão de recursos humanos, dentro das empresas, parece necessitar atuar de forma inovadora e precisa, com vistas a alcançar condições de competitividade. Isto porque esta área surge como núcleo vital e estratégico para assegurar o correto funcionamento da empresa, principalmente no setor de serviços, uma vez que sua principal tarefa, consiste em cuidar de seu capital mais valioso.

\section{As POLÍTICAS DE RECURSOS IIUMANOS NA $\mathbb{E} \mathbb{M} \mathbb{P} \mathbb{R} \mathbb{E} \mathbb{A}$ COMPETITIVA}

A concorrência está no âmago do sucesso ou do fracasso das empresas, determinando a adequação das atividades que podem contribuir para o seu desempenho, como inovações, uma cultura coesa ou uma boa implementação. A estratégia competitiva é a busca de uma posição competitiva favorável em uma indústria, a arena fundamental onde ocorre a concorrência (PORTER, 1998).

LADO e WILSON (1994) notaram que a empresa pode gerar vantagem competitiva através da criação concomitante de comportamentos e valores que ajudam a proporcionar valores agregados para os clientes. Usar a gestão de recursos humanos como fonte de vantagem competitiva requer que a empresa não reaja, simplesmente, aos desafios do meio ambiente, mas antecipe e explore a área de competitividade futura (BEER eä AL, 1985; HENDRY e PETTIGREW, 1992; GUEST, 1987; GENNARD e KELLY, 1994; LADO e WILSON, 1994; CAPELLI e CROFTERHEFTER, 1996).

A gerência de recursos humanos pode afetar a vantagem competitiva em qualquer empresa, através de seu papel na determinação das qualificações e da motivação dos empregados e do custo de contratação e do treinamento. Em alguns segmentos ela é a base para a vantagem competitiva (PORTER, 1998). No caso específico das empresas de serviços, parece ficar mais evidente que as políticas e práticas de recursos humanos estabelecidas aparecem como fundamentais para dar suporte à obtenção e manutenção dessa vantagem competitiva, já que o fator humano é condição essencial para o resultado do serviço prestado.

FISHER (1998) aponta um novo significado assumido pela administração de recursos humanos como vantagem competitiva. Com a perspectiva voltada para o negócio da empresa, começa a ser levantado o papel das pessoas para que se consiga competitividade. Se a estratégia é a diferenciação e a obtenção de vantagem competitiva, cabe a administração de recursos humanos garantir que as pessoas sejam fonte de vantagem competitiva. As empresas precisam buscar formas novas e criativas de gestão; à administração de recursos humanos cabe transmitir estratégias às pessoas e transformá-las em agentes de competitividade.

ALBUQUERQUE (1999) propõe algumas premissas relacionadas às políticas de recursos humanos, que coloca como necessárias para o enquadramento das empresas aos novos padrões incluídos na proposta de organização competitiva:

"valorização dos talentos humanos na empresa; atração e manutenção de pessoas de alto potencial e qualificação para o trabalho; criação de condições favoráveis à motivação individual e à mobilização dos grupos em torno das metas organizacionais; possibilidades de crescimento funcional e desenvolvimento profissional na própria empresa; oferecimento de incentivos vinculados a resultados da atividade empresarial e adapta- 
ção de políticas integradas de recursos humanos à realidade da empresa e ao contexto externo econômico-social e político na qual atua" (p.232).

As políticas e práticas de recursos humanos devem criar organizações que sejam melhores equipadas para executar estratégias, operar com eficiência, envolver os funcionários e gerenciar a mudança, em se tratando de elementos da organização competitiva (ULRICH, 1998).

Para as empresas que pretendam um melhor posicionamento no mercado, parece, então, necessária a definição de formas novas relacionadas às políticas e práticas de recursos humanos, para a obtenção de resultados efetivos em termos de inovações, qualidade dos serviços e produtividade no trabalho.

Transformar a força de trabalho passou a ser o maior desafio estratégico enfrentado pelas empresas que esperam ter sucesso. E como a transformação das pessoas dá-se através da educação, o desafio é, principalmente, de educação e reeducação de todos os níveis e grupos dentro das empresas (GONÇALVES, 1997). Ainda de acordo com GONÇALVES (1997):

"a agenda de transformação das pessoas que tripularão as empresas do futuro inclui: como desenvolver e gerenciar o pessoal para os novos papéis que deverão assumir; como utilizar novos modelos de avaliação de desempenho, remuneração e educação do pessoal; como criar uma organização em que cada pessoa esteja voltada à criação de valor para o cliente; como saber empregar técnicas para reformular a cultura, adequando-a ao novo mundo dos negócios" ( pg.17).

As organizações de serviços podem tornar-se mais eficientes e eficazes investindo continuamente no funcionário, utilizando programas de reconhecimento e remuneração e respeitando as individualidades. Entretanto, essas medidas devem ser vistas como um investimento em pessoas, melhorando a comunicação, dando poderes aos funcionários, estimulando uma gestão participativa, estabelecendo programas de capacitação, e não como um custo, já que elas trazem, a médio e longo prazos, resultados efetivos para a performance da empresa.

\section{O P $\mathbb{R O C E S S O ~ D O ~} \mathbb{M} \mathbb{A} \mathbb{R} \mathbb{R} \mathbb{E} \mathbb{N} \mathbb{N} \mathbb{I} \mathbb{N} \mathbb{E} \mathbb{R} O$}

Nas organizações de hospitalidade, os empregados distribuem os produtos e, através desta distribuição, eles se tornam, no sentido já mencionado, parte destes produtos. Expressando esse ponto de vista, KOTLER et AL. (1999) colocam que o hotel precisa ter um grupo de empregados que atue bem no ato da prestação dos serviços. Portanto, os esforços de marketing devem ser direcionados, primeiramente, para os clientes internos, que se constituem no primeiro mercado para a organização.

Dentro desse contexto, GEORGE e GRÖNROOS 1991) consideram o marketing interno como uma filosofia para gerenciar os recursos humanos da organização com base em uma perspectiva do marketing. Essa filosfia deve ser transformada em um processo continuo, a fim de que a motivação duradoura para orientação ao cliente e ao serviço seja atingida entre os empregados. O marketing interno usa a perspectiva do marketing para gerenciar os empregados de uma empresa (GRÖNROOS, 1990).

Para PIERCY e MORGAN (1991), a proposta básica que eles chamam de marketing interno consiste no desenvolvimento de um programa de marketing dirigido para os funcionários da companhia, que é paralelo e coerente com o programa de marketing para o mercado externo formado pelos clientes e concorrentes.

Dessa forma, os autores sugerem que as mesmas técnicas de comunicação e análise que são utilizadas para abordar o mercado externo deveriam ser adaptadas e usadas para difundir as estratégias e planos para objetivos importantes dentro da organização, conforme mostra a Figura 1. 
Figura 1: Plano de Marketing

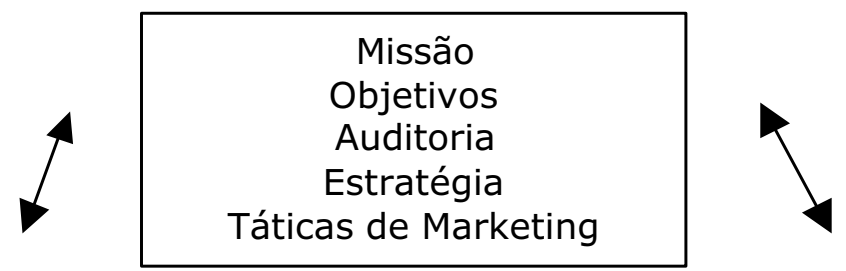

\begin{tabular}{|l|}
\hline \multicolumn{1}{|c|}{$\begin{array}{c}\text { Programa de } \\
\text { Marketing Externo }\end{array}$} \\
\hline $\begin{array}{l}\text { Produto } \\
\text { Preço } \\
\text { Comunicação } \\
\text { Distribuição }\end{array}$ \\
\multicolumn{1}{|c|}{} \\
GRUPOS-ALVO-CHAVE \\
PARA A COMPANHIA
\end{tabular}

\begin{tabular}{|l|}
\hline \multicolumn{1}{|c|}{$\begin{array}{c}\text { Programa de } \\
\text { Marketing Interno }\end{array}$} \\
\hline $\begin{array}{l}\text { Produto } \\
\text { Preço } \\
\text { Comunicação } \\
\text { Distribuição }\end{array}$ \\
\multicolumn{1}{|c}{} \\
SEGMENTOS DE \\
CLIENTES-CHAVE
\end{tabular}

FONTE: PIERCY, N E MORGAN, N. Internal Marketing - The Missing Half of the Marketing Program, Journal of Long Range Planning, vol.24, p.84, 1991.

Ainda os autores PIERCY e MORGAN (1991) desenvolvem uma estrutura de um programa de marketing interno que pode ser apresentado dentro dos seguintes termos:

- Produto: no nível mais elementar, "produto" corresponde às estratégias e ao plano de marketing no qual elas estão contidas. Contudo, o que está implícito é que o produto a "ser vendido" é composto pelos valores, atitudes e comportamentos que são necessários para fazer o plano de marketing funcionar;

- Preço: refere-se ao valor que os clientes internos devem "pagar" quando eles compram o produto e o plano de marketing. Isto pode incluir o sacrifício de outros projetos que competem ao plano de marketing, mas, fundamentalmente, refere-se ao custo psicológico a ser pago pela adoção de valores-chave diferentes e da mudança da forma como os trabalhos são realizados.

- Comunicação: o aspecto mais tangível do programa de marketing interno é a mídia da comunicação, usada para informar, persuadir e trabalhar as atitudes do pessoal chave no mercado interno. Isto inclui comunicações por escrito, tais como resumos do plano e relatórios, mas também apresentações públicas para grupos ou indivíduos que são importantes para o sucesso do plano;

- Distribuição: este elemento está preocupado com os lugares físicos e sócio-técnicos no qual devem ser entregues os produtos e suas comunicações: reuniões, comitês, sessões de treinamento para gerentes e pessoal de assessoria, seminários, relatórios, comunicações.

A partir desta estrutura, pode-se verificar que, talvez, o canal real de distribuição esteja no gerenciamento de recursos humanos, no treinamento, na avaliação e sistemas de recompensas, no recrutamento voltado para a captação de perfis compatíveis com os valores das estratégias de marketing, de tal modo que a cultura da organização torna-se o canal de distribuição para estratégias de marketing interno.

No que se refere ao produto do marketing interno, GRÖNROOS (1990) aponta que a organização precisa oferecer a seu público interno de empregados um "produto" que seja atrativo. Produto este que pode ser traduzido como um trabaIho e ambiente que motivem os empregados a responderem favoravelmente às demandas do gerenciamento. 
A qualidade do produto "emprego" dependerá, entre outras coisas, dos métodos de gerenciamento, da política de pessoal, da natureza do trabalho e do planejamento e execução dos processos. Deste modo, o "produto" deve ser desenvolvido com cuidado e orientado como os produtos externos • serviços, requerendo, para tanto, uma decisão estratégica para o gerenciamento relacionado com estes assuntos.

Nesse sentido, o engajamento dos recursos humanos é necessário para que o marketing interno se torne bem sucedido. Entende-se que o sucesso das atividades de marketing interno deve ser alcançado quando a empresa assegure que seus empregados estejam motivados para atingir uma performance orientada para o cliente e ou serviço e, ao mesmo tempo, sintam-se dispostos a permanecer como membros da organização.

O marketing interno, enquanto filosofia gerencial e por meio de suas ferramentas, tem como objetivo desenvolver no empregado um compromisso com o serviço e com o cliente.

\section{O IIOTEL OBIETO DO ESTUDO}

O hotel estudado pertence a um grupo de serviços empresariais com 17 (dezessete) marcas nas áreas de Alimentação e Restaurantes, Hotelaria e Viagens, Produtividade e Marketing. Em 1999, contava com um total de 20 mil funcionários e uma receita operacional de US $\$ 3,8$ bilhões. Abrange vários países, formando-se assim uma extensa diversificação horizontal que configura os conglomerados multinacionais.

O hotel está situado na Região Metropolitana do Recife, a $15 \mathrm{Km}$ do centro da cidade e a $5 \mathrm{~km}$ do aeroporto. Localizado à beira mar, conta com 108 (cento e oito) apartamentos, com serviço de quarto, serviço de lavanderia, estacionamento, restaurante, bar, áreas para eventos e área de lazer (piscina). Abriga ainda, em suas dependências, o Departamento Regional (Norte/Nordeste) de Recursos Humanos para o Setor de Hotelaria, que está diretamente vinculado à matriz do grupo.

Configura-se, portanto, como um hotel de médio porte. Sua estrutura está voltada ao atendimento de uma clientela formada, principalmente, por executivos e público de eventos.

O grupo hoteleiro apresenta, como resumo do papel da organização no mercado e na sociedade, o que ele denomina de Projeto de Empresa no Brasil, baseado em três eixos (People, Service, Profit). No eixo People, o grupo acredita que as pessoas podem realizar seus sonhos a partir da empresa, numa relação de parceria; o eixo Service representa o jeito da empresa de servir e o Profit, a possibilidade dos colaboradores lucrarem junto com a empresa.

Partindo dessas premissas, a política que norteia a área de Recursos Humanos do Grupo baseia-se em dez pontos principais: escolhendo as pessoas; integrando as pessoas; criando condições para as pessoas se sentirem motivadas; construindo e mantendo equipes; lidando com as divergências; acompanhando o desempenho; treinando as pessoas; realizando a missão da empresa; garantindo a qualidade organizacional e criando uma qualidade de vida para as pessoas (MANUAL DE RECURSOS HUMANOS DO GRUPO, 2000).

\section{ASPECTOS MIETODOLÓGICOS}

A presente pesquisa caracteriza-se como qualitativa de caráter exploratório, sendo identificada como um estudo de caso. O estudo de caso tem sido aplicado quando o foco de interesse é sobre fenômenos atuais analisados dentro de um contexto de vida real e que, portanto, responde às questões "como" e "por quê" certos fenômenos ocorrem (GODOY, 1995). 
A população da pesquisa constituiu-se de 57 (cinqüenta e sete) colaboradores, representando todos os níveis hierárquicos da organização. O tipo de amostragem utilizada foi a não probabilística, selecionada por tipicidade, tendo sido constituída por elementos considerados representativos da população-alvo.

A amostra da pesquisa foi dividida em grupos formados pelos colaboradores do hotel, totalizando 45 (quarenta e cinco) pessoas, contemplando-se assim cinco grupos, três dos quais pertencentes ao organograma do hotel em estudo e dois às gerências regionais de recursos humanos e vendas, conforme Tabela 1.

Tabela 1: Amostra da pesquisa

\begin{tabular}{lc}
\hline \multicolumn{1}{c}{ ÁREA } & No EMPREGADOS \\
\hline Gerência Geral & 01 \\
Média Chefia & 07 \\
Operacional & 35 \\
Gerência Regional de RH & 01 \\
Gerência Regional de Vendas & 01 \\
\hline T OTAL & $\mathbf{4 5}$ \\
\hline
\end{tabular}

Como técnica fundamental da pesquisa qualitativa, foram realizadas entrevistas, do tipo semi-estruturadas, através de roteiros, diferenciados em duas categorias, uma direcionada para as gerências regionais do grupo e gerência geral do hotel e outra para as demais categorias funcionais da empresa.

Para dar suporte ao estudo, foram coletados dados secundários, obtidos através de pesquisa documental realizada junto à empresa, tendo sido examinados: relatórios, pesquisas internas/externas, manuais, publicações, comunicações internas, o site do grupo (na Internet) e documentos (registros, correspondências, ofícios, memorandos) que foram reexaminados, recebendo tratamento analítico e que puderam complementar o estudo.

Para a interpretação dos dados obtidos, utilizou-se a metodologia denominada análise de conteúdo (BARDIN, 1979), tomando-se como referência a revisão de literatura constante deste trabalho.

\section{$\mathbb{P}_{\mathbb{R} I N C I P A I S} \mathbb{R} \mathbb{E} S U L T A D O S$}

Através da pesquisa, constatou-se que a gestão de recursos humanos adotada pelo hotel objeto deste estudo, apresenta-se consistente e bem estruturada, o que permite criar condições para despertar e desenvolver no seu colaborador o comprometimento com a qualidade na prestação dos serviços hoteleiros.

Os resultados permitiram identificar que o hotel possui um programa de marketing interno que é coerente com o esforço de marketing externo, pois o hotel procura aplicar as técnicas de comunicação e análise para o mercado externo, semelhantes àquelas utilizadas para o mercado interno. Nesse sentido, é oferecido aos colaboradores um programa de marketing, baseado na oferta do produto emprego, no custo psicológico desse emprego, na distribuição desse produto e na promoção que a empresa se utiliza para difundir sua estratégia corporativa.

O hotel apresenta uma estrutura organizacional enxuta o que permite a atuação mais rápida e objetiva dos colaboradores que estão em contato direto com o cliente. Os poucos níveis hierárquicos possibilitam um trabalho compartilhado e, ao mesmo tempo, solicitam das chefias o desempenho de um papel muito mais de liderança, de orientação e de parceria para o trabalho.

Quanto às políticas e práticas de recursos humanos, verificou-se, de forma geral, que a maioria dos funcionários as consideram satisfatórias. A empresa oferece um conjunto de vantagens formado por salário, benefícios, remuneração variável, investimento em treinamento e participação nos lucros. São atrativos que fazem os funcionários optarem por permanecer no emprego, principalmente quando comparado ao que é oferecido pelos demais hotéis integrantes do mercado local. 
Algumas ressalvas devem ser feitas no que diz respeito ao item remuneração, em que alguns dos colaboradores não se mostraram plenamente satisfeitos, mas que, dentre estes, a maioria consegue perceber que é o próprio mercado que estabelece os baixos salários, característicos da hotelaria na região. Outro ponto a considerar é que simplesmente o fato de existir a possibilidade de participação nos lucros, proposto pela empresa, não se constitui um incentivo de peso, na medida que o colaborador parece ver como uma possibilidade distante, já que não recebe esses resultados há três anos.

Com relação à estrutura do sistema de comunicação do hotel, os resultados obtidos demonstraram haver uma circulação de informações, entre chefes e subordinados, entre colegas de trabalho e entre os diversos departamentos, que favorece o bom desempenho das atividades e também colabora para sedimentar as normas e valores compartilhados pela empresa e, portanto, reforça a cultura da empresa.

Essas normas e valores são estabelecidos e disseminados dentre os colaboradores do hotel através de vários mecanismos, como reuniões, treinamentos, sistemas de comunicação, documentos. Verificou-se também, haver uma identificação e/ou o compromisso dos colaboradores com esses valores e normas, o que vem a ser corroborado, pelo tempo de permanência no emprego da maioria dos funcionários, pois $64,4 \%$ têm entre um e dez anos de hotel, o que é um dado relevante já que na hotelaria como um todo se verifica um alto grau de rotatividade.

Um outro ponto a considerar, é o fato da maioria dos colaboradores, em todos os níveis, ter a convicção do seu papel e de suas atividades no desempenho do trabalho e no atendimento, com qualidade, ao hóspede. Isso pode ser o reflexo de sua consciência e compromisso com as normas e valores do hotel.

O treinamento em serviços é um outro fator de extrema relevância no contexto da formação e solidificação do compromisso com a qualidade na prestação dos serviços, pois, principalmente, através dele são repassados os requisitos relacionados aos conhecimentos e atitudes para a realização de um serviço de qualidade. Pôde-se detectar, através dos resultados, que a empresa possui uma política de treinamento bastante consistente, com vários tipos de cursos que estão voltados para os diversos cargos, funções e níveis hierárquicos.

Esses treinamentos são dados dentro da empresa, através da gerente de recursos humanos ou das médias chefias, que são preparadas com cursos internos e/ou externos. Com relação ao nível gerencial, a Universidade Institucional realiza freqüentemente cursos em diversas áreas à medida da necessidade.

Diante dos resultados e considerações apresentadas pôde-se verificar como um hotel pode conseguir, através do investimento na gestão de recursos humanos, criar uma consciência e um compromisso voltados para o cliente externo e para a prestação de um serviço de qualidade, pré-requisito imprescindível para uma empresa que pretenda ser ou tornar-se competitiva.

\section{CONSIDERAÇÕES IFINAIS}

Os resultados do estudo permitiram constatar que o hotel possui uma cultura organizacional forte e que consegue estabelecer uma relação de parceria com a maioria de seus colaboradores, a qual lhes permite realizar um trabalho em equipe, participativo e comprometido com o atendimento ao hóspede. Entretanto, apesar de seguir as tendências positivas para gestão de recursos humanos, o hotel precisa, constantemente, estar em processo de reavaliação de suas estratégias, políticas e práticas de recursos humanos, a fim de que possa estar sempre em condições de competitividade.

Apesar da hotelaria ainda trabalhar, de um modo geral, com salários reduzidos e um contingente significativo de pessoas com baixo nível educacional, verificase que o hotel oferece, aos seus colaboradores, um conjunto de benefícios e oportunidades de crescimento que o diferencia daquilo que pode ser considerado como 
regra na indústria hoteleira. Essas características fazem do grupo e, especificamente, do hotel em estudo, um local que apresenta uma cultura organizacional que o faz caracterizar-se como uma empresa que oferece condições para que o seu colaborador seja consciente do cliente e orientado para a qualidade do serviço.

Em contrapartida, essa não parece representar a regra para o setor hoteleiro, constituído, na sua maioria, por empresas familiares e que ainda não demonstram estar conscientes da necessidade de investir nas pessoas, para a obtenção do retorno desejado, bem como garantir sua permanência no mercado, de forma competitiva, frente aos grandes grupos hoteleiros que começam a se instalar no Brasil e, particularmente, em Pernambuco.

A partir dessas considerações, vale ressaltar as limitações deste estudo, que tem relação com a complexidade e a dimensão do tema escolhido, o que suscita um aprofundamento do estudo da área de recursos humanos em hotéis, passando a temas mais específicos, relacionados às suas políticas e práticas.

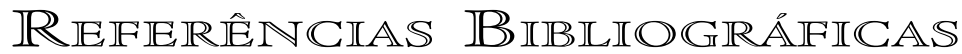

ALBUQUERQUE, L. G. (1999)."Estratégias de Recursos Humanos e Competitividade", in: VIEIRA, M. M. F. \& OLIVEIRA, L. M. B. Administração Contemporânea. São Paulo: Atlas (p.215-238).

BARDIN, Laurence. Análise de conteúdo. Lisboa.Edições 70. 1979.

BASSI, Eduardo. Globalização de Negócios. São Paulo: Cultura Editores Associados, 1997.

BEER, M. SPECTOR, B. LAWRENCE, P. MILLS, D. WALTON, R. Human Resource Management: a general manager's perspective. New York: Free Press. 1985.

BOOG, G.G. Manual de Treinamento e Desenvolvimento - ABDT - Associação Brasileira de Treinamento e Desenvolvimento. São Paulo: Makron Books. 1994.

CAPELLI, P., CROFTER-HEFTER, A. Distinctive human resources are firms core competencies. Organization Dynamics, vol. 24, n. 3 p. 7-22. 1996.

CARVALHO, Virgílio N. da S. Por uma política de recursos humanos. Revista Turis em Análise, ECA-USP. São Paulo. V. 2, n. 2, p. 14-23, nov. 1991.

COBRA, Marcos e RANGEL, Alexandre. Serviços ao cliente: uma estratégia competitiva. São Paulo: Marcos Cobra Editora, 1992.

FISHER, André. A constituição do modelo competitivo de gestão de pessoas no Brasil: um estudo sobre as empresas consideradas exemplares. Tese de Doutoramento, Faculdade de Economia Administração e contabilidade da Universidade de São Paulo, 1998.

GENNARD, J., KELLY, J. Human resource management: the views of personnel directors. Human Resource Management Journal, v. 5, n. 1, p.15-32, 1994.

GEORGE, W. R., GRONROOS, Christian. Developing Customer Conscious Employees at every level: internal marketing. New York: American Management Association. 1991.

GODOY, Arilda Schimidt. Introdução à pesquisa qualitativa e suas possibilidades. RAE - Revista de Administração de Empresas. São Paulo, v. 35, n.2, p 57-63, 1995.

GONÇALVES, J. E. L. Os novos desafios da empresa do futuro. RAE. Revista de Administração de Empresas. São Paulo: FVG, v. 37, n. 3, p. 10-19, jul/set. 1997.

GRONROOS, C. Service Management and marketing. Lexington, Mass: Lexington Books, 1990. 
GURPO ACCOR-NHT. Manual de Recursos Humanos - Como selecionar, acolher, integrar e desenvolver-se na NHT Hotelaria e Turismo. São Paulo. 2000.

GUEST D. E. Human resource management and industrial relations. Journal of Management Studies, v. 24, n. 503-521, 1987.

HENDRY, C. , PETTIGREW, A. Patterns of strategic change in the development of human resource management. British Journal of Management, v. 3, p. 137-156, 1992.

KOTLER, P. BOWEN, J. MAKENS, J. Marketing hospitality and tourism. 2 ed. NJEUA: Prentice-Hall, 1999.

LADO, A A , WILSON, M. Human resource systems and sustained competitive advantage: a competence based perspective. Academy of Management Review, v. 19, n.4, p 699-727, 1994.

MARQUARDT, M. J., ENGEL, D. W. Global human resource management. Englewood. Chifs: Prentice-Hall, 1993.

PANORAMA Setorial/Gazeta Mercantil.Análise Setorial: a indústria hoteleira. LUZ, M.C.V. São Paulo. Vol. 1, abr. 1999.

PIERCY, N., MORGAN, N. Internal marketing - the missing half of the marketing programme. Journal of Long Range Planning, 24 (2), p. 82-93. 1991.

PORTER, Michael. Seminário "Reinventing Strategy", HSM, São Paulo, 6/12/94.

PORTER, Michael.Vantagem competitiva: criando e sustentando um desempenho superior. $11^{\text {a }}$ ed. Rio de Janeiro: Campus. 1998.

SCHULER, R. S., HUBER, V. L. Personnel and human resource management. USA: West Publishing Company, fifith edition, 1993.

ULRICH, David. Os campeões de recursos humanos: inovando para obter meIhores resultados. São Paulo: Futura, 1998. 